\title{
Exenatide enhances INS-1 rat pancreatic $\beta$-cell mass by increasing the protein levels of adiponectin and reducing the levels of $\mathrm{C}$-reactive protein
}

\author{
HUIBIAO QUAN $^{1}$, YONGYI GAO ${ }^{1}$, HUACHUAN ZHANG ${ }^{1}$, TUANYU FANG ${ }^{1}$, \\ DAOXIONG CHEN ${ }^{1}$, ZHAOHUI LV $^{1}$ and YANMING CHEN ${ }^{2}$
}

${ }^{1}$ Department of Endocrinology, People's Hospital of Hainan Province, Haikou, Hainan 570311; ${ }^{2}$ Department of Endocrinology, The Third Affiliated Hospital of Sun Yat-Sen University, Guangzhou, Guangdong 510630, P.R. China

Received August 14, 2013; Accepted May 16, 2014

DOI: $10.3892 / \mathrm{mmr} .2014 .2538$

\begin{abstract}
Type 2 diabetes mellitus (T2DM) is a complex and heterogeneous disorder affecting $>220$ million individuals worldwide; this is projected to reach 366 million by 2030 . Exenatide, a long-acting glucagon-like peptide 1 receptor agonist, exhibits potential in the treatment of T2MD due to its ability to increase $\beta$-cell mass. However, the molecular mechanism by which exenatide increases $\beta$-cell mass is yet to be elucidated. Exenatide function was explored in the INS-1 rat pancreatic $\beta$-cell line. Exenatide was found to increase adiponectin protein levels by $20 \%(\mathrm{P}<0.05$ versus the control group) and reduce the level of C-reactive protein (CRP) by $50 \%$ ( $\mathrm{P}<0.01$ versus the control group) in INS-1 cells, resulting in an increase in the INS-1 rat pancreatic $\beta$-cell mass by $20 \%$ $(\mathrm{P}<0.01$ versus the control group). These findings suggest that exenatide may ameliorate T2DM by increasing adiponectin protein levels and reducing the level of CRP.
\end{abstract}

\section{Introduction}

Type 2 diabetes mellitus (T2DM) is a complex and heterogeneous disorder affecting >220 million individuals worldwide; this is projected to reach 366 million by 2030 (1). Despite the number of individuals affected by the disease, the therapeutic strategies that are currently available for T2DM are limited. These strategies involve insulin and four main classes of oral antidiabetic agents that act to i) stimulate the secretion of insulin by the pancreas, such as sulfonylureas, rapid-acting secretagogues/insulinotropic agents, glipizide, glibenclamide and rapaglinide; ii) reduce the production of glucose by the

Correspondence to: Dr Huibiao Quan, Department of Endocrinology, People's Hospital of Hainan Province, Building 6, 19 Xiuhua Road, Haikou, Hainan 570311, P.R. China

E-mail: huibiaohn@163.com

Key words: exenatide, adiponectin, C-reactive protein, INS-1 rat pancreatic $\beta$-cell line liver, including biguanides (metformin); iii) delay the digestion and absorption of carbohydrate in the intestine, including $\alpha$-glucosidase inhibitors and acarbose; or iv) enhance the action of insulin, including thiazolidinediones, pioglitazone and rosiglitazone. However, none of these agents is completely effective (2).

Numerous pharmaceutical studies have focused on the development of novel drugs to reduce the symptoms associated with the long-term complications of diabetes (3-5). Glucagon-like peptide-1 (GLP-1) is an incretin hormone of the enteroinsular axis. In healthy individuals, GLP-1 is secreted subsequent to eating, and lowers glucose concentrations by increasing insulin secretion and suppressing glucagon release. Furthermore, GLP-1 impairs gastric emptying, suppresses appetite and has been suggested to inhibit $\beta$-cell apoptosis (6). However, native GLP-1 is degraded within 2-3 min in the circulation (6). Therefore, a number of GLP-1 receptor agonists have been developed to prolong the in vivo effect of GLP-1. Exenatide, a GLP-1 receptor agonist that exhibits sustained activity, has potential for the treatment of T2DM due to its ability to enhance insulin secretion and increase $\beta$-cell mass (7-9). Although the preparation and antidiabetic activity of exenatide have been widely reported $(10,11)$, the molecular mechanism by which exenatide improves $\beta$-cell mass is yet to be elucidated.

Experimental evidence from animals and healthy subjects indicates that GLP-1 may be involved in the control of appetite and the intake of energy in patients with T2DM $(12,13)$. Furthermore, GLP-1 has been suggested as a potential treatment for T2DM due to its effects on glycemic control, insulin sensitivity and $\beta$-cell function (14). $\beta$ cells function to promote insulin production and maintain glucose homeostasis (15), which is critical for the prevention and treatment of T2DM. Adiponectin is a fat-derived hormone that is known to exhibit antidiabetic and anti-atherogenic effects. Adiponectin acts to stimulate the uptake of glucose into skeletal muscle cells via the activation of insulin receptor substrate-1-mediated phosphatidylinositol-3 kinase function, suppress the production of glucose by the liver and enhance $\beta$-oxidation in the muscle via the activation of $5^{\prime}$ adenosine monophosphate-kinase (16). C-reactive protein (CRP) has been shown to be an independent 
predictor of risk for the development of diabetes (17). However, it has been reported that, in patients with T2DM, exenatide treatment has durable and persistent beneficial effects on CRP (18). In addition, exenatide increases adiponectin levels in patients with T2DM (19). Thus, we hypothesized that exenatide could enhance $\beta$-cell proliferation through an adiponectin-induced reduction in $\beta$-cell apoptosis (20) and a reduction in the levels of CRP, a sign of inflammation and potential risk in patients with T2DM (17).

\section{Materials and methods}

Plasmid constructs, antibodies and reagents. Exenatide is a single, non-glycosylated peptide containing 39 amino acids (HGEGTFTSDLSKQMEEEAVRLFIEWLKNGGPSSGAP

PPS) and has a molecular mass of 4,186.6 Da. Full-length genes of exenatide and adiponectin were constructed by overlap extension polymerase chain reaction (PCR), followed by the subcloning of the constructs into various vectors. Histidine (His)-tag exenatide was expressed in BL21 (DE3) E. coli and purified using Ni-nitrilotriacetic acid resin. Anti-Myc antibody (1 mg/ml; Clontech Laboratories Inc. Mountain View, CA, USA) was used for the assay of the results of the co-immunoprecipitation (Co-IP) test. Anti-Flag monoclonal antibody (1 mg/ml; Sigma-Aldrich, St. Louis, MO, USA) and anti-green fluorescent protein (GFP) antibody (2 mg/ml; Cell Signaling Technology, Inc., Danvers, MA, USA) were used for fluorescence staining. Anti-exenatide and -adiponectin polyclonal antibodies $(1 \mathrm{mg} / \mathrm{ml}$; Abcam, Cambridge, UK) were used for western blot analysis. Anti-glutathione S-transferase (GST; $0.5 \mathrm{mg} / \mathrm{ml}$ ) and -His $(0.1 \mathrm{mg} / \mathrm{ml})$ antibodies were used for the assay of the results of the GST-pull-down analysis, and were purchased from Tiangen Biotech Co. Ltd. (Beijing, China). GAPDH (1 $\mathrm{mg} / \mathrm{ml})$ and secondary antibodies $(1 \mathrm{mg} / \mathrm{ml})$ were purchased from Santa Cruz Biotechnology, Inc. (Santa Cruz, CA, USA). Various vectors were amplified in E. coli, isolated using a QIAprep ${ }^{\circledR}$ Miniprep kit (Qiagen, Inc., Chatsworth, CA, USA) and verified by automated DNA sequencing. Anti-CRP antibody (1 mg/ml; Shengshi Zhongfang BioSci $\&$ Tech) was used for western blot analysis. $\beta$-actin antibody was purchased from Abcam. The present study was approved by the ethics committee of the People's Hospital of Hainan Province (Haikou, China).

Rat INS-1 cell culture and incubation. INS-1 cells were purchased from the Shanghai Cell Bank (Shanghai, China). INS-1 cells were grown in monolayer cultures in RPMI-1640 medium at $37^{\circ} \mathrm{C}$ in a humidified atmosphere, with $5 \% \mathrm{CO}_{2}$ and $95 \%$ air. INS-1 cells were harvested and divided into three groups. The cells in the three groups were exposed to normal concentrations of glucose $(5 \mathrm{mM})$, high concentrations of glucose $(30 \mathrm{mM})$ or high concentrations of glucose (30 mM) plus exenatide (100 $\mathrm{nM})$, for $24 \mathrm{~h}$ each. The levels of adiponectin and CRP and INS-1 cell proliferation were determined after three days of culturing. INS-1 cell proliferation was determined by direct cell counting. For direct cell counting, $5 \times 10^{4}$ cells were seeded and harvested after three days of culture, then counted using a hemacytometer (Hausser Scientific, Horsham, PA, USA).
Quantitative (q)PCR. Total RNA was isolated from cells using QIAshredder and RNeasy ${ }^{\circledR}$ Mini kits (Qiagen, Inc.). cDNA was synthesized using $500 \mathrm{ng}$ RNA extracts in a volume of $20 \mu \mathrm{l}$ using Avian Myeloblastosis Virus reverse transcriptase XL (Takara Bio Inc., Dalian, China) priming with random 9-mers at $42^{\circ} \mathrm{C}$ for $10 \mathrm{~min}$. The cDNA was stored at $20^{\circ} \mathrm{C}$ until use. qPCR was performed using $\mathrm{SYBR}^{\circledR}$-Green I Master Mix in the Light-Cycler ${ }^{\circledR} 480$ System (Roche, Mannheim, Germany). RNA was isolated from non-transfected and transfected INS-1 cells, followed by cDNA synthesis and data analysis as described previously (9). The primers for qPCR were as follows: Adiponectin, 5'-GTCCTAAGGGAGACATCG GTG-3' (forward) and 5'-CCATACACCTGGAGCCAGAC-3' (reverse); CRP, 5'-CTGTCCTCGACCCGTGGGTAC-3' (forward) and 5'-CTGGTGACAGCACAAAGTC-3' (reverse) and GAPDH, 5'-CCCTTCATTGACCTCAACTAC-3' (forward) and 5'-CCACCTTCTTGATGTCATCAT-3' (reverse). GAPDH was used as an internal control. The AmpliTaq Gold ${ }^{\circledR}$ enzyme was activated by heating for $10 \mathrm{~min}$ at $95^{\circ} \mathrm{C}$, and all genes were amplified by 50 cycles of heating for $15 \mathrm{sec}$ at $95^{\circ} \mathrm{C}$, followed by $1 \mathrm{~min}$ at $60^{\circ} \mathrm{C}$.

For the construction of standard curves of positive controls, the total RNA of primary neuronal cells was reverse-transcribed into cDNA and serially diluted in water in five or six log steps to generate four-fold serial dilutions of cDNA between $100 \mathrm{pg}$ and $100 \mathrm{ng}$. These cDNA serial dilutions were stored at $20^{\circ} \mathrm{C}$. The coefficient of linear regression was calculated for each standard curve, and using the cycle threshold value for each sample, the relative concentration of adiponectin, CRP and GAPDH were calculated. To normalize for differences in the quantity of total RNA in each starting reaction, GAPDH expression was used as an endogenous control. The data represent the average expression of target genes, relative to GAPDH, from three independent cultures.

Transfection of INS-1 cells and western blot analysis. The INS-1 cells ( $2 \times 10^{5} / \mathrm{p}-35$ plate) were transfected with various vectors. Transfection was performed in 50-60\% confluent cells in plates using $9 \mu 1$ Lipofectamine 2000 ${ }^{\mathrm{TM}}$ (Applied Biosystems, Foster City, CA, USA). Forty-eight hours subsequent to transfection, the cells were split and clones were selected for antibiotic resistance. Resistant colonies were either pooled or cloned by ring isolation.

All transfected and non-transfected cells were homogenized in radioimmunoprecipitation assay buffer $[150 \mathrm{mM}$ $\mathrm{NaCl}, 1 \%$ Nonidet P-40 (NP-40), $0.5 \%$ sodium deoxycholate, $0.1 \% \mathrm{SDS}$ and $50 \mathrm{mM}$ Tris- $\mathrm{HCl}(\mathrm{pH}$ 8.0)] with Complete Mini Protease Inhibitor (Roche). Following debris removal, the supernatants were boiled and mixed with an equal volume of $20 \%$ glycerol containing $0.02 \%$ bromophenol blue. Proteins were separated by SDS-PAGE and transferred to a polyvinylidene difluoride membrane (Millipore, Billerica, MA, USA). The membranes were blocked with $5 \%$ skimmed milk in $10 \mathrm{mM}$ Tris $(\mathrm{pH} 7.5), 100 \mathrm{mM} \mathrm{NaCl}$ and $0.1 \%$ Tween-20 (TBST) and incubated with primary antibodies in TBST with $0.5 \%$ skimmed milk overnight at $4^{\circ} \mathrm{C}$. The membrane was treated with primary antibodies and horseradish peroxidase-conjugated goat anti-mouse immunoglobulin G secondary antibodies, diluted 1:3,000 (Amersham Biosciences, Amersham, UK). Immunoreactive 
bands were visualized by enhanced chemiluminescence (GE Healthcare, Amersham, UK) and quantified by densitometry using ImageJ 1.45 software (National Institutes of Health, Bethesda, MD, USA) according to the manufacturer's instructions.

Fluorescence microscopy. At 24 h post-transfection, the cells were fixed with $2 \%$ paraformaldehyde for $10 \mathrm{~min}$, prior to washing with phosphate-buffered saline (PBS). The cells were subsequently permeabilized using $1 \%$ Triton X-100 for $10 \mathrm{~min}$, washed with PBS and incubated with monoclonal antibodies for $1 \mathrm{~h}$, followed by incubation with secondary antibodies for $1 \mathrm{~h}$. The cell nuclei were stained with $0.1 \mathrm{~g} / \mathrm{ml}$ DAPI and the cells were observed using a fluorescence microscope.

Immunoprecipitation. The cells were harvested and lysed in $20 \mathrm{mM}$ HEPES (pH 7.2), $50 \mathrm{mM} \mathrm{NaCl}, 0.5 \%$ Triton X-100, $1 \mathrm{mM} \mathrm{NaF}$ and $1 \mathrm{mM}$ dithiothreitol (HEPES lysis buffer). The lysate was incubated with the indicated antibodies for $3 \mathrm{~h}$ at $4^{\circ} \mathrm{C}$, prior to the addition of protein $\mathrm{A} / \mathrm{G}$-plus agarose. Immunoprecipitates were washed three times in lysis buffer, and analyzed using western blotting.

GST pull-down assay. Bacteria-expressed GST or GST-adiponectin proteins were immobilized on glutathione-Sepharose 4B beads (Amersham) and washed. The beads were then incubated with exenatide and washed with GST binding buffer $(100 \mathrm{mM} \mathrm{NaCl}, 50 \mathrm{mM} \mathrm{NaF}, 2 \mathrm{mM}$ EDTA and $1 \%$ NP-40). The proteins were subsequently eluted, prior to use in western blot analysis.

RNA interference (RNAi). Small interfering (si)RNA directed againstadiponectin(5'-GTTGCTGGGAGCTGTTCTACT-3'), CRP (5'-GAG TCGGATACTTCCTATGTA-3') and non-target control siRNA (5'-UUCUCCGAACGUGUCACGU-3') were synthesized by Shanghai GenePharm Co., Ltd. (Shanghai, China).

Statistical analysis. All results are presented as the mean \pm standard deviation. Student's unpaired t-tests were performed for the comparison of individual data and two-way analysis of variance with Fisher's protected least significant difference post hoc tests was performed for repeated measures over time. $\chi^{2}$ tests were performed to compare differences in distribution. A value of $\mathrm{P}<0.05$ was considered to indicate statistical significance.

\section{Results}

Relative adiponectin and CRP $m$ RNA levels in INS-1 cells. qPCR analysis revealed that the mRNA levels of adiponectin were significantly reduced and those of CRP significantly increased in the INS-1 cells in the $30 \mathrm{mM}$ group, compared with those in the $5 \mathrm{mM}$ group. However, upon the addition of exenatide, mRNA levels of adiponectin were found to increase and those of CRP decrease in the cells in the $30 \mathrm{mM}$ group (Fig. 1). These results suggest that exenatide can increase the mRNA levels of adiponectin and reduce those of CRP in INS-1 cells.

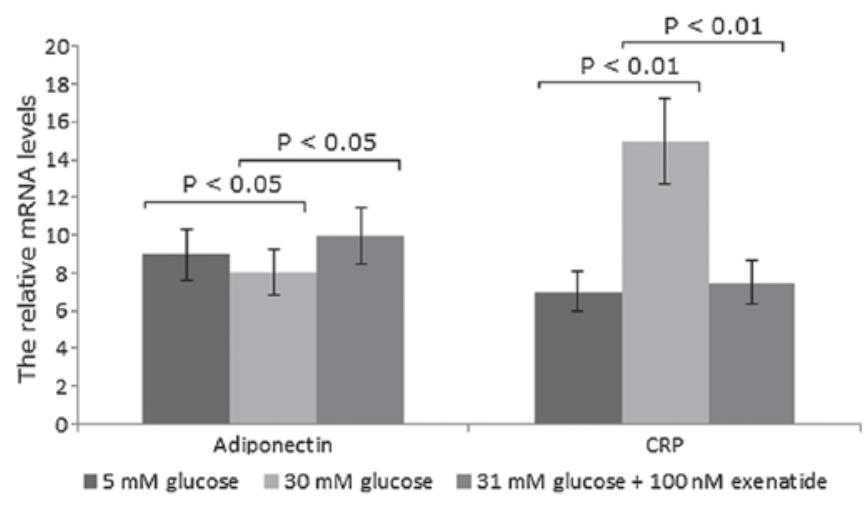

Figure 1. Quantitative polymerase chain reaction analysis of adiponectin and CRP mRNA levels in rat INS-1 cells after three days of culture. The INS-1 cells were divided into three groups and exposed to normal concentrations of glucose $(5 \mathrm{mM})$, high concentrations of glucose $(30 \mathrm{mM})$ or high concentrations of glucose plus exenatide ( $30 \mathrm{mM}$ glucose and $100 \mathrm{nM}$ exenatide) for $24 \mathrm{~h}$ each. Relative mRNA levels were normalized to levels of GAPDH. Each bar represents the mean \pm standard deviation of three independent experiments. CRP, C-reactive protein.
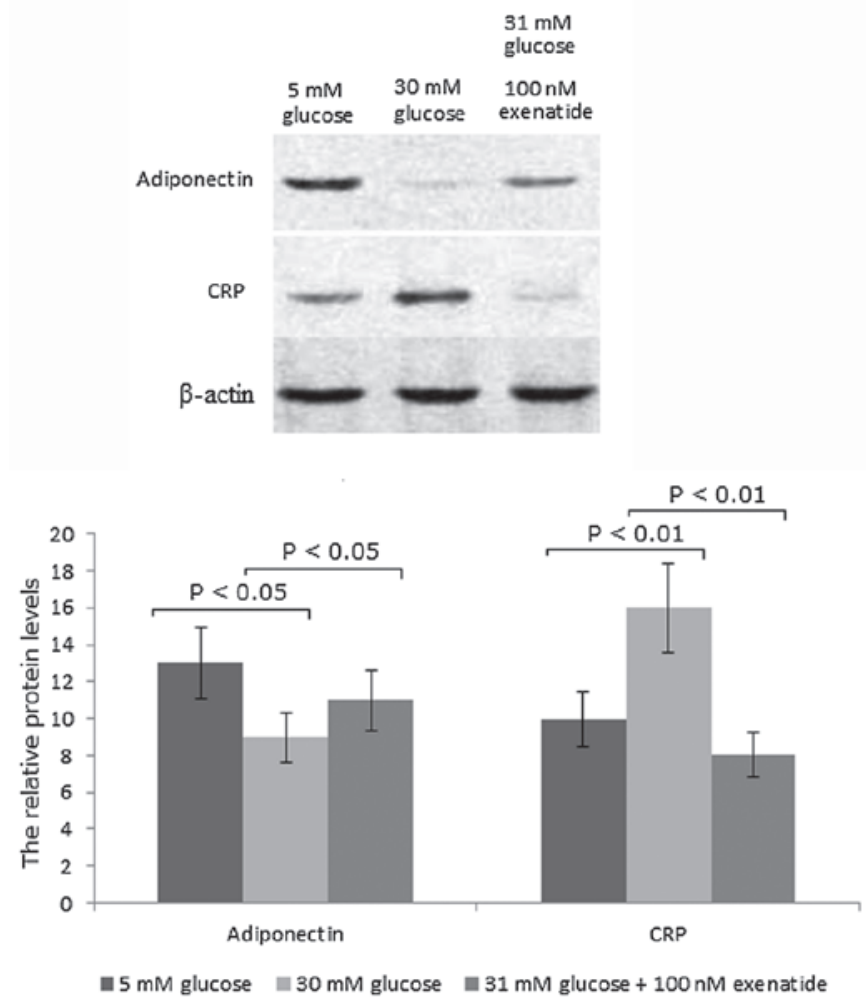

Figure 2. Western blot analysis of adiponectin and CRP protein levels in rat INS-1 cells after three days of culture. The INS-1 cells were divided into three groups and exposed to normal concentrations of glucose $(5 \mathrm{mM})$, high concentrations of glucose $(30 \mathrm{mM})$ or high concentrations of glucos plus exenatide (30 mM glucose and $100 \mathrm{nM}$ exenatide) for $24 \mathrm{~h}$ each. Relative mRNA levels were normalized to levels of GAPDH. Each bar represents the mean \pm standard deviation of three independent experiments. CRP, C-reactive protein.

Adiponectin and CRP protein levels in INS-1 cells. In accordance with the qPCR results, western blot analysis revealed that the protein levels of adiponectin were reduced and those of CRP were increased in INS-1 cells in the $30 \mathrm{mM}$ group compared with those in the $5 \mathrm{mM}$ group. However, upon the 
A

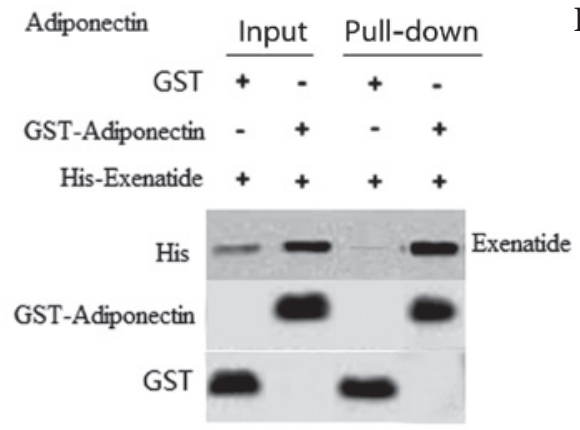

B

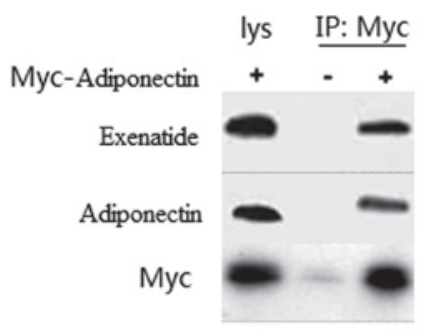

C

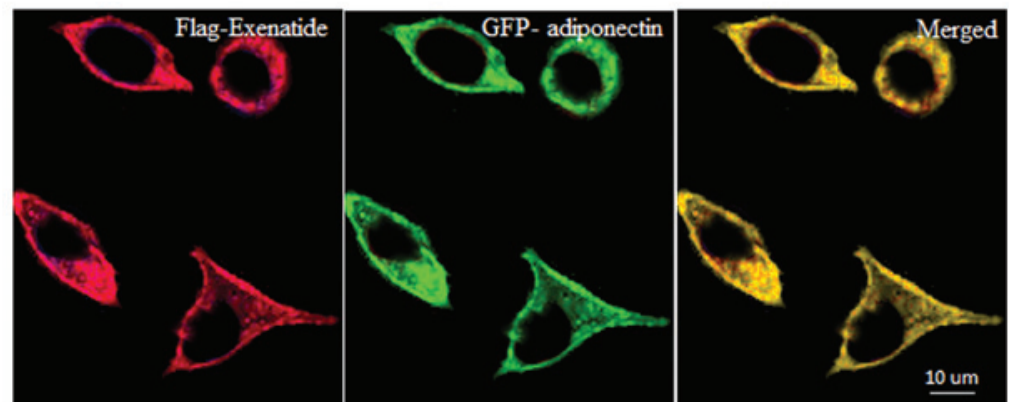

Figure 3. Interaction between exenatide and adiponectin. (A) Direct interaction between exenatide and adiponectin was revealed by GST pull-down assays Input and pull-down samples were subjected to immunoblotting using anti-GST and anti-His antibodies. The input represents $10 \%$ of that used for the pull-down assay. (B) Co-immunoprecipitation of exenatide and adiponectin in INS-1 cells. Cell lysates were immunoprecipitated using anti-Myc antibody and analyzed by immunoblotting. (C) Exenatide and adiponectin co-localize in the membrane of INS-1 cells. GST, glutathione S-transferase; His, histidine; IP, immunoprecipitation; GFP, green fluorescent protein; lys, lysate.

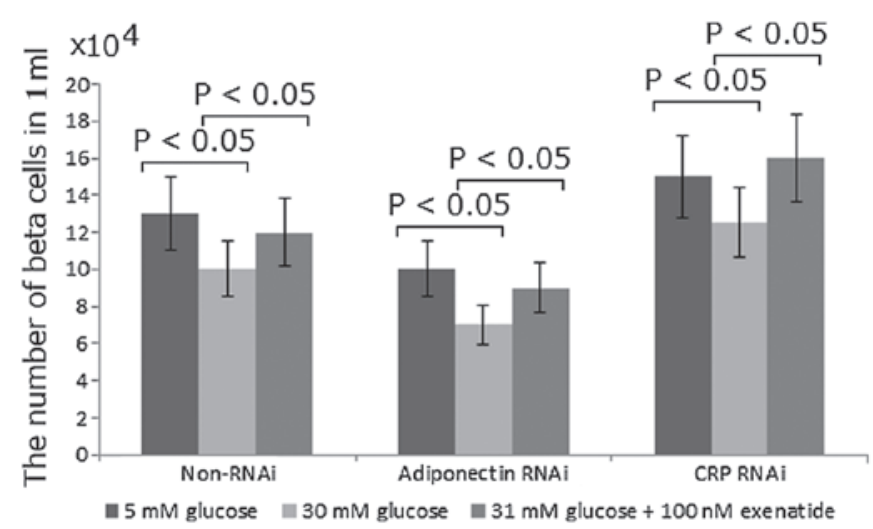

Figure 4. Effect of adiponectin and CRP on $\beta$-cell number following three days of culture. The number of $\beta$ cells was determined by direct cell counting on day three after culturing. INS-1 cells were divided into three groups. The cells in the three groups were exposed to normal concentrations of glucose $(5 \mathrm{mM})$, high concentrations of glucose $(30 \mathrm{mM})$ or high concentrations of glucose plus exenatide ( $30 \mathrm{mM}$ glucose and $100 \mathrm{nM}$ exenatide) for $24 \mathrm{~h}$ each. INS-1 cells were further divided into three groups for RNAi treatment: Non-RNAi, adiponectin RNAi and CRP RNAi. Each bar represents the mean \pm standard deviation of three independent experiments. CRP, C-reactive protein; RNAi, RNA interference.

addition of exenatide, the protein levels of adiponectin were increased by $20 \%(\mathrm{P}<0.05)$ and those of CRP were reduced by $50 \%(\mathrm{P}<0.01)$ in the cells in the $30 \mathrm{mM}$ group (Fig. 2). These findings suggest that exenatide can increase the protein levels of adiponectin and reduce those of CRP in INS-1 cells.

Exenatide interacts with adiponectin. To determine the interaction between exenatide and adiponectin, in vitro GST pull-down assays using recombinant GST-adiponectin and
His-exenatide were performed. A specific interaction was observed between exenatide and adiponectin, but not with GST alone (Fig. 3A). To assess whether exenatide interacts with adiponectin, a Co-IP assay was performed in INS-1 cells. The Co-IP results further revealed an association between exenatide and adiponectin (Fig. 3B), which suggests that these two proteins may co-localize. To assess the subcellular localization of exenatide and adiponectin, INS-1 cells were transfected with GFP-adiponectin and Flag-exenatide. When coexpressed, exenatide and adiponectin were observed to be co-localized in the membrane of the INS-1 cells (Fig. 3C).

Exenatide enhances the INS-1 rat pancreatic $\beta$-cell mass by increasing the protein levels of adiponectin and reducing the levels of $C R P$. It has previously been reported that exenatide treatment may activate adiponectin protein expression, which may have a protective effect against $\beta$-cell apoptosis (20). In the present study, to determine whether adiponectin could increase $\beta$-cell mass, the function of adiponectin was inhibited using RNAi. Compared with the non-RNAi group, the number of $\beta$ cells was reduced by $20 \%$ with RNAi treatment (Fig. 4). By contrast, compared with the non-RNAi group, the inhibition of CRP using RNAi was observed to increase the number of $\beta$ cells by $15 \%$ (Fig. 4). In all the cases, high concentrations of glucose were found to reduce the number of $\beta$ cells while exenatide was capable of correcting the change by increasing the number of $\beta$ cells (Fig. 4). Exenatide treatment was observed to increase the number of $\beta$ cells by $20 \%$. These results suggest that exenatide may improve the INS-1 rat pancreatic $\beta$-cell mass by increasing the protein levels of adiponectin and reducing the level of CRP. 


\section{Discussion}

In the present study, compared with the control group, the levels of CRP were found to be significantly increased in the high-dose glucose group while those of adiponectin were reduced. Reduced levels of adiponectin and high levels of CRP are frequently followed by a decrease in the number of $\beta$ cells. Adiponectin acts against cytokine- and fatty acid-induced $\beta$-cell apoptosis and completely restores the function of insulin-producing cells that have been disrupted by autoimmunity and lipotoxicity (21). In addition, CRP is closely associated with obesity in T2DM (22), while free fatty acids in obesity may cause $\beta$-cell dysfunction and depletion (23).

It has previously been reported that high glucose levels can induce apoptosis in cultured human pancreatic islets (24). High concentrations of glucose may elevate the level of oxidative stress in INS-1 cells, leading to an increase in apoptosis and a decrease in proliferation. It has been suggested that exenatide may have a protective effect on INS-1 cells exposed to high-glucose environments. In the present study, exenatide was observed to be capable of reversing the increase in CRP and the decrease in adiponectin levels in the high-dose glucose group; therefore, exenatide may enhance INS-1 rat pancreatic $\beta$-cell mass by increasing the protein levels of adiponectin and reducing the levels of CRP.

Exenatide monotherapy has been of clinical interest in patients with T2DM due to reported improvements in glycemic control and weight with the use of exenatide in combination with oral antidiabetic agents $(25,26)$. However, its limitations include dose-dependent glucoregulatory activity and dose-limiting nausea and vomiting. A gradual escalation in the dose of exenatide can reduce the incidence of dose-limiting nausea and vomiting, without a concurrent loss of glucoregulatory activity; therefore, gradual dose-escalation may be beneficial for attenuating the gastrointestinal side-effects of exenatide (27). However, exenatide therapy is also limited by the relatively short half-life of the drug (28). It has been reported that modified exenatide, a synthetic form of exendin-4, which is a glucagon-like peptide hormone that regulates insulin secretion, has a native half-life of $2.4 \mathrm{~h}$ and shows a projected half-life of $139 \mathrm{~h}$ (29). Therefore, expressing novel modified exenatide in various eukaryotic systems will be the focus of our future investigations.

In conclusion, the present study demonstrated that Exenatide can increase adiponectin protein levels and reduce the level of CRP in INS-1 cells, resulting in an increase in INS-1 rat pancreatic $\beta$-cell mass. These findings suggest that Exenatide may ameliorate T2DM by increasing adiponectin protein levels and reducing the level of CRP.

\section{References}

1. Anvari M: Use of metabolic surgery for the treatment of type 2 diabetes. Can J Diabetes 35: 99-108, 2011.

2. Srinivasan K and Ramarao P: Animal models in type 2 diabetes research: an overview. Indian J Med Res 125: 451-472, 2007.

3. Bennett WL, Maruthur NM, Singh S, et al: Comparative effectiveness and safety of medications for type 2 diabetes: an update including new drugs and 2-drug combinations. Ann Intern Med 154: 602-613, 2011.

4. Pathania S, Randhawa V and Bagler G: Prospecting for novel plant-derived molecules of Rauvolfia serpentina as inhibitors of Aldose Reductase, a potent drug target for diabetes and its complications. PloS one 8: e61327, 2013.
5. Sugimoto T and Kashiwagi A: The cutting-edge of medicine; novel therapeutic agents for the treatment of diabetes sodium-glucose co-transporter (SGLT) 2 inhibitors. Nihon Naika Gakkai Zasshi 102: 1474-1483, 2013 (In Japanese).

6. Meier JJ: GLP-1 receptor agonists for individualized treatment of type 2 diabetes mellitus. Nat Rev Endocrinol 8: 728-742, 2012 .

7. Gedulin BR, Nikoulina SE, Smith PA, et al: Exenatide (exendin-4) improves insulin sensitivity and \{beta\}-cell mass in insulin-resistant obese $\mathrm{fa} / \mathrm{fa}$ Zucker rats independent of glycemia and body weight. Endocrinology 146: 2069-2076, 2005.

8. Bunck MC, Diamant M, Cornér A, et al: One-year treatment with exenatide improves beta-cell function, compared with insulin glargine, in metformin-treated type 2 diabetic patients: a randomized, controlled trial. Diabetes Care 32: 762-768, 2009.

9. Fehse F, Trautmann M, Holst JJ, et al: Exenatide augments firstand second-phase insulin secretion in response to intravenous glucose in subjects with type 2 diabetes. J Clin Endocrinol Metab 90: 5991-5997, 2005.

10. Tripathy NR, Basha S, Jain R, Shetty S and Ramachandran A: Exenatide and acute pancreatitis. J Assoc Physicians India 56: 987-988, 2008

11. Kendall DM, Riddle MC, Rosenstock J, et al: Effects of exenatide (exendin-4) on glycemic control over 30 weeks in patients with type 2 diabetes treated with metformin and a sulfonylurea. Diabetes Care 28: 1083-1091, 2005.

12. Gutzwiller JP, Drewe J, Göke B, et al: Glucagon-like peptide-1 promotes satiety and reduces food intake in patients with diabetes mellitus type 2. Am J Physiol 276: R1541-R1544, 1999.

13. Zander M, Madsbad S, Madsen JL and Holst JJ: Effect of 6-week course of glucagon-like peptide 1 on glycaemic control, insulin sensitivity, and beta-cell function in type 2 diabetes: a parallel-group study. Lancet 359: 824-830, 2002.

14. Navarro M, Rodriquez de Fonseca F, Alvarez E, et al: Colocalization of glucagon-like peptide-1 (GLP-1) receptors, glucose transporter GLUT-2, and glucokinase mRNAs in rat hypothalamic cells: evidence for a role of GLP-1 receptor agonists as an inhibitory signal for food and water intake. J Neurochem 67: 1982-1991, 1996.

15. Nir T, Melton DA and Dor Y: Recovery from diabetes in mice by beta cell regeneration. J Clin Invest 117: 2553-2561, 2007.

16. Iwaki M, Matsuda M, Maeda N, et al: Induction of adiponectin, a fat-derived antidiabetic and antiatherogenic factor, by nuclear receptors. Diabetes 52: 1655-1663, 2003.

17. Freeman DJ, Norrie J, Caslake MJ, et al: C-reactive protein is an independent predictor of risk for the development of diabetes in the West of Scotland Coronary Prevention Study. Diabetes 51: 1596-1600, 2002.

18. Bergenstal RM, Wysham C, Macconell L, et al; DURATION-2 Study Group: Efficacy and safety of exenatide once weekly versus sitagliptin or pioglitazone as an adjunct to metformin for treatment of type 2 diabetes (DURATION-2): a randomised trial. Lancet 376: 431-439, 2010.

19. Derosa G, Putignano P, Bossi AC, et al: Exenatide or glimepiride added to metformin on metabolic control and on insulin resistance in type 2 diabetic patients. Eur J Pharmacol 666: 251-256, 2011.

20. Wijesekara N, Krishnamurthy M, Bhattacharjee A, Suhail A, Sweeney G and Wheeler MB: Adiponectin-induced ERK and Akt phosphorylation protects against pancreatic beta cell apoptosis and increases insulin gene expression and secretion. J Biol Chem 285: 33623-33631, 2010.

21. Rakatzi I, Mueller H, Ritzeler O, Tennagels $\mathrm{N}$ and Eckel J: Adiponectin counteracts cytokine-and fatty acid-induced apoptosis in the pancreatic beta-cell line INS-1. Diabetologia 47: 249-258, 2004.

22. Kahn SE, Zinman B, Haffner SM, et al; ADOPT Study Group: Obesity is a major determinant of the association of $\mathrm{C}$-reactive protein levels and the metabolic syndrome in type 2 diabetes. Diabetes 55: 2357-2364, 2006.

23. Boden G and Shulman GI: Free fatty acids in obesity and type 2 diabetes: defining their role in the development of insulin resistance and beta-cell dysfunction. Eur J Clin Invest 32 (Suppl 3): 14-23, 2002.

24. Federici M, Hribal M, Perego L, et al: High glucose causes apoptosis in cultured human pancreatic islets of Langerhans: a potential role for regulation of specific Bcl family genes toward an apoptotic cell death program. Diabetes 50: 1290-1301, 2001. 
25. Moretto TJ, Milton DR, Ridge TD, et al: Efficacy and tolerability of exenatide monotherapy over 24 weeks in antidiabetic drug-naive patients with type 2 diabetes: a randomized, double-blind, placebo-controlled, parallel-group study. Clin Ther 30: 1448-1460, 2008.

26. Gentilella R, Bianchi C, Rossi A and Rotella CM: Exenatide: a review from pharmacology to clinical practice. Diabetes Obes Metab 11: 544-556, 2009.

27. Fineman MS, Shen LZ, Taylor K, Kim DD and Baron AD: Effectiveness of progressive dose-escalation of exenatide (exendin-4) in reducing dose-limiting side effects in subjects with type 2 diabetes. Diabetes Metab Res Rev 20: 411-417, 2004.
28. Brubaker PL: Incretin-based therapies: mimetics versus protease inhibitors. Trends Endocrinol Metab 18: 240-245, 2007.

29. Chen C, Constantinou A and Deonarain M: Modulating antibody pharmacokinetics using hydrophilic polymers. Expert Opin Drug Deliv 8: 1221-1236, 2011. 